Supporting Information for

\title{
Continuous Flow Composite Membrane Catalysts for Efficient Decomposition of Chemical Warfare Agent Simulants
}

Jin Young Seo ${ }^{a, b, \dagger}$, Kie Yong Cho ${ }^{a, c, \dagger}$, Jung-Hyun Lee ${ }^{b}$, Min Wook Leed, ${ }^{d,}$, and Kyung-Youl Baek ${ }^{a, e, f^{*}}$

a. Materials Architecturing Research Center, Korea Institute of Science and Technology, Seoul 02792, Republic of Korea.

b. Department of Chemical and Biological Engineering, Korea University, Seoul 136-713, Republic of Korea.

c. Department of Industrial Chemistry, Pukyong National University, Pusan 48547, Republic of Korea.

d. Institute of Advanced Composite Materials, Korea Institute of Science and Technology, Jeonbuk 55324, Republic of Korea.

e. Division of Nano \& Information Technology, KIST School, Korea University of Science and Technology, Seoul 02792, Republic of Korea.

f. Center for Convergent Chemical Process, Korea Research Institute of Chemical Technology, Daejeon 34114, Republic of Korea.

t These authors contributed equally to this work.

*Corresponding author:mwlee0713@kist.re.kr, baek@kist.re.kr

Keywords: chemical warfare agents (CWAs), metal-organic frameworks (MOFs), nanofibers, composite membranes, hydrolysis reactions 

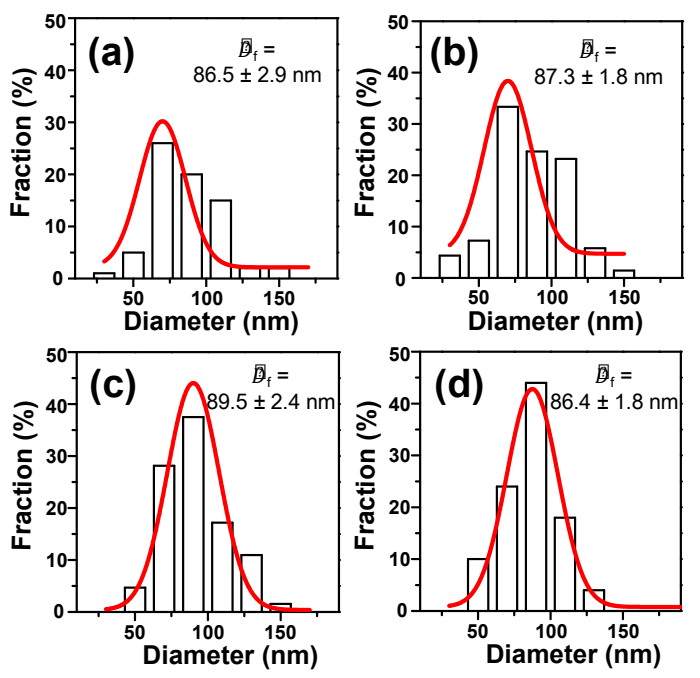

Figure S1. Nylon 6 nanofiber diameter distribution of (a) nylon 6 nanofibers, (b) CMC33,

(c) CMC46, and (d) CMC56 ( $\bar{D}_{\mathrm{f}}=$ average diameter of electrospun nanofibers).
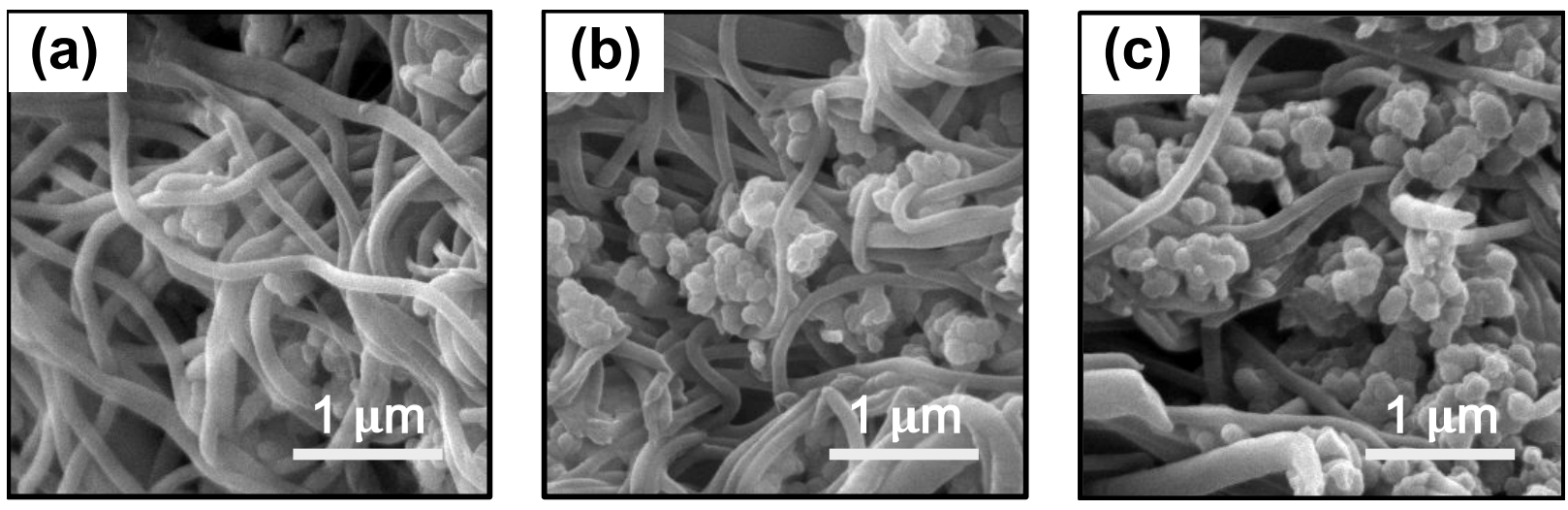

Figure S2. Cross-sectional SEM images (a) CMC33, (b) CMC46, and (c) CMC56. 
(a)

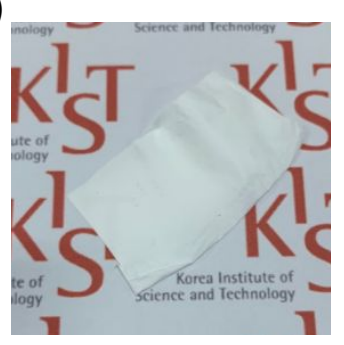

(b)

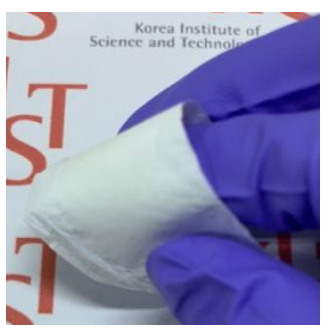

Figure S3. Visual image of (a) CMC56 and (b) its bending image.

Calculation of UiO-66 weight fraction in UiO-66-nanofiber from TGA data.

The weight fraction of UiO-66 in each CMC was calculated from TGA analysis by comparing the mass of residue UiO-66 which was used in this paper was assumed as $\mathrm{Zr}_{6} \mathrm{O}_{4}(\mathrm{OH})_{4}\left(\mathrm{C}_{8} \mathrm{H}_{4} \mathrm{O}_{4}\right)_{4.2}\left[\left(\mathrm{H}_{2} \mathrm{O}\right)(\mathrm{OH})\right]_{3.6}$ by following previous reported paper. [1] When UiO66 was heated up to $800^{\circ} \mathrm{C}$ under $\mathrm{N}_{2}$ atmosphere, carbon and metal from UiO-66 were remained. On the other hands, nylon 6 was fully removed at $800{ }^{\circ} \mathrm{C}, \mathrm{CMC}$ sample's remained weight was considered as carbon and zirconium from UiO-66. The following equation is used to determine the amount of UiO-66 in each $\mathrm{CMC}$ based on remaining amounts at $800^{\circ} \mathrm{C}$. 


$$
\begin{aligned}
& =\left((\text { Mass of zirconium \& carbon in CMC })\left(\frac{\text { mass of UiO }-66}{\text { mass of zirconium and carbon in UiO }-66}\right)\right. \\
& =\left(\left(\text { Mass of remain of } \mathrm{CMC} @ 800^{\circ} \mathrm{C}\right) *\left(\frac{\text { mass of } \mathrm{UiO}-66}{\text { mass of remain of } \mathrm{UiO}-66 @ 800^{\circ} \mathrm{C}}\right)\right.
\end{aligned}
$$

Once the weight of UiO-66 in CMC was determined, the weight fraction of UiO-66 in CMC

can be calculated by comparing with a total mass of CMC.

$$
\text { Weight Fraction of UiO-66 in CMC }=\frac{\text { Weight of UiO }-66 \text { in CMC }}{\text { Weight of CMC }}
$$


Table S1. $\mathrm{Zr}$ contents of UiO-66, CMCs, and nylon 6, and UiO-66 weight fraction in composites.

\begin{tabular}{ccccc} 
Entry & Sample Name & $\begin{array}{c}\text { Zr contents }^{\text {a }} \\
\left(\mathrm{mmol} \mathrm{g}^{-1}\right)\end{array}$ & $\begin{array}{c}\text { UiO-66 Fraction a } \\
(\%)\end{array}$ & $\begin{array}{c}\text { UiO-66 Fraction b } \\
\text { b }\end{array}$ \\
\hline $\mathbf{1}$ & UiO-66 & 2.83 & 100 & 100 \\
$\mathbf{2}$ & CMC33 & 0.92 & 32.5 & 33 \\
$\mathbf{3}$ & CMC46 & 1.29 & 45.5 & 46 \\
$\mathbf{4}$ & CMC56 & 1.55 & 54.7 & 56 \\
$\mathbf{5}$ & Nylon 6 & - & 0 & 0
\end{tabular}

a the UiO-66 fraction calculated by ICP, b UiO-66 fraction evaluated by TGA.

Table S2. The actual and ideal surface area, and Catalytically Active Surface of CMCs

with different UiO-66 loading amounts.

\begin{tabular}{ccccc} 
Entry & Sample Name & $\begin{array}{c}\mathrm{SA}_{\text {ideal }}{ }^{\mathrm{a}} \\
\left(\mathrm{m}^{2} \mathbf{g}^{-1}\right)\end{array}$ & $\begin{array}{c}\mathrm{SA}_{\text {actual }} \\
\left(\mathbf{m}^{2} \mathbf{~ g}^{-1}\right)\end{array}$ & $\begin{array}{c}\text { Catalytically Active Surface } \\
(\%)\end{array}$ \\
\hline 1 & CMC33 & 513.9 & 354 & 68.8 \\
2 & CMC46 & 699.0 & 420 & 60.0 \\
3 & CMC56 & 841.4 & 585 & 69.5
\end{tabular}

a The ideal surface area was calculated using $\left(S A_{\mathrm{UiO}}-66 \mathrm{X} W_{\mathrm{UiO}-66}+S A_{\text {Nylon6 }} \times W_{\text {Nylon 6 }}\right)$ (whereas $S A_{\mathrm{a}}$, and $W_{\mathrm{a}}$ means surface area and weight fraction of component a respectively.) ${ }^{\mathrm{b}}$ The catalytically active surface was evaluated using ( $S A_{\text {actual }} / S A_{\text {ideal }} \mathrm{X}$ 100) 


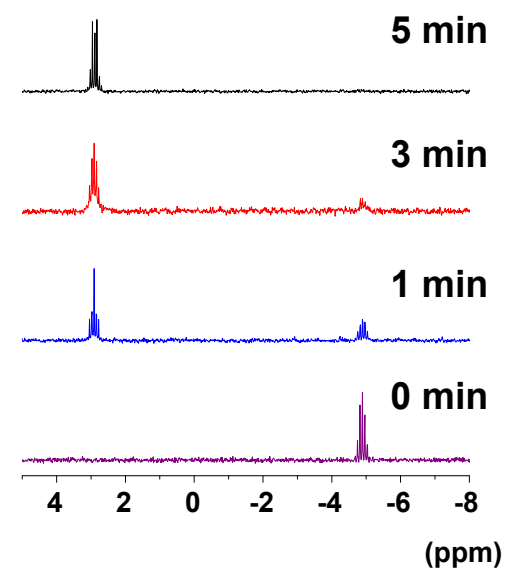

Figure S4. The ${ }^{31} \mathrm{P}$ NMR spectra of MPO hydrolysis reaction solution with CMC56.

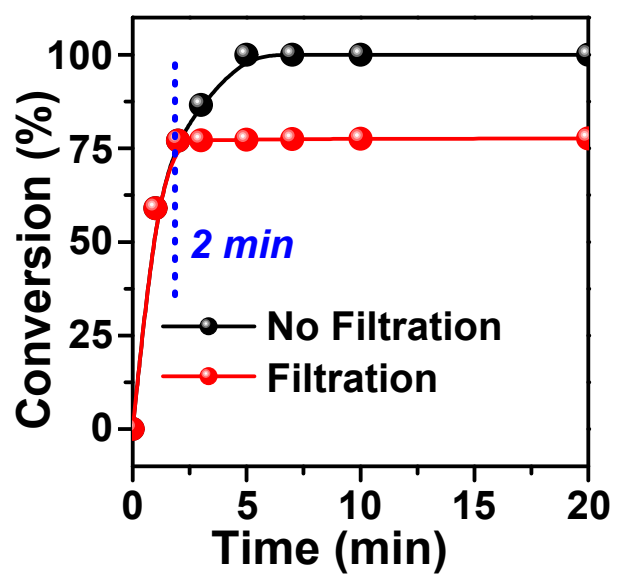

Figure S5. Hydrolysis profile of MPO under filtering test with CMC56.

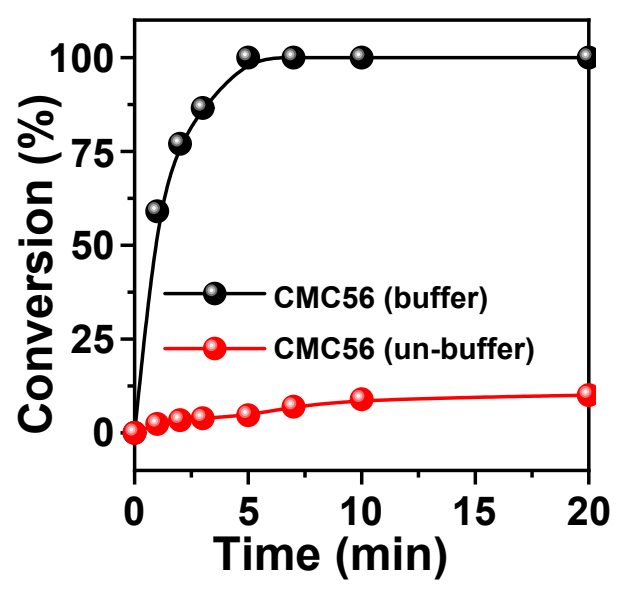


Figure S6. MPO hydrolysis conversion vs time plot using CMC56 with and without N-EM buffer.

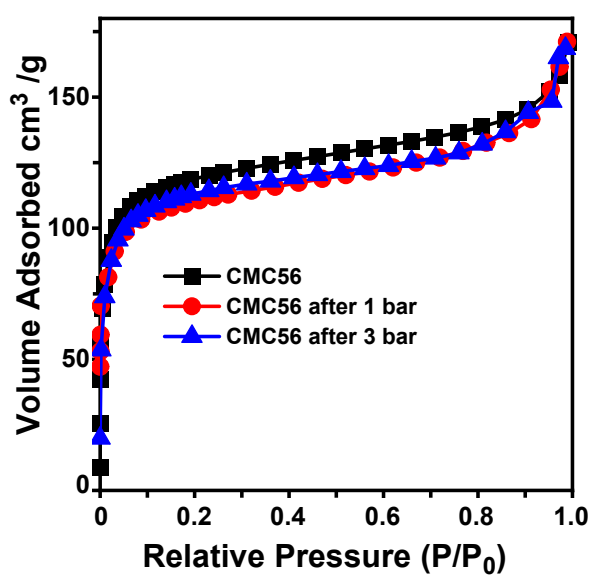

Figure S7. $\mathrm{N}_{2}$ adsorption isotherm analysis of CMC56 after exposure to hydraulic pressure for $24 \mathrm{hr}$.

(a)

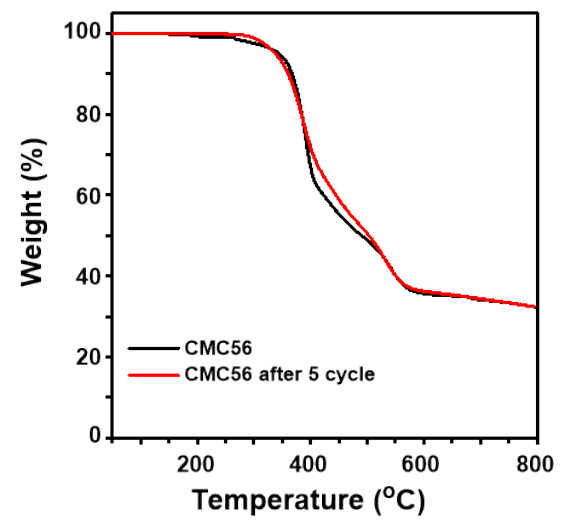

(b)

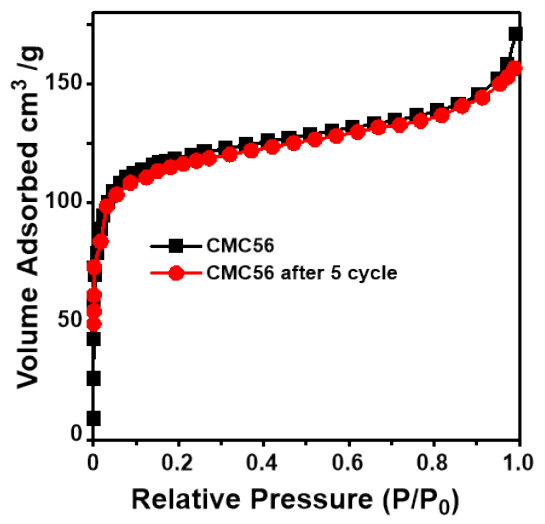

(c)

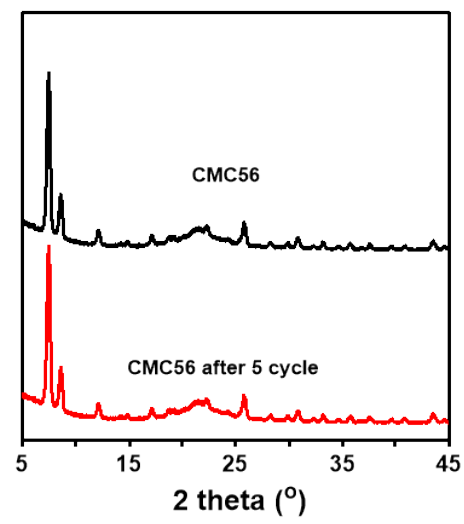

Figure S8. Characterization of CMC56 before, and after 5 cycle of MPO hydrolysis (a) TGA under $\mathrm{N}_{2}$; (b) $\mathrm{N}_{2}$ adsorption isotherm; and (c) XRD patterns. 


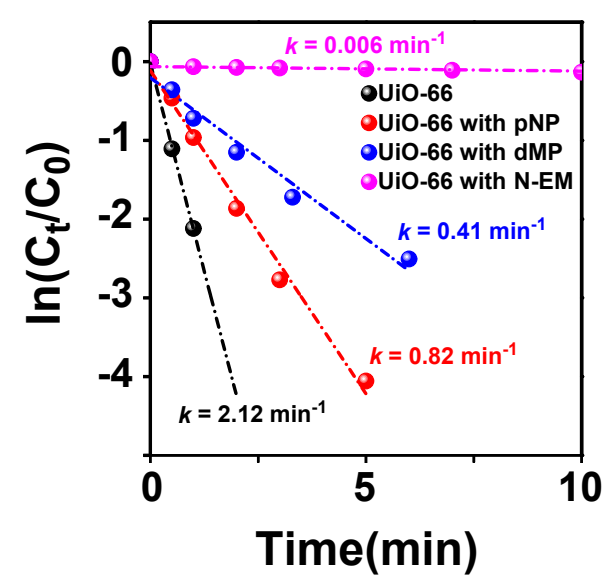

Figure S9. The $\ln \left(C_{t} / C_{0}\right)$ vs. time plots for hydrolysis of MPO with contaminated UiO-66.

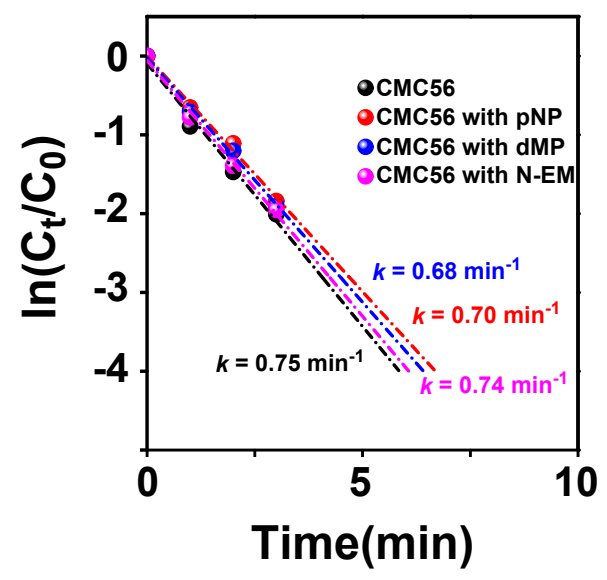

Figure S10. The $\ln \left(C_{t} / C_{0}\right)$ vs. time plots for hydrolysis of MPO with contaminated CMCs.

\section{Reference}

(1) Cho, K. Y.; Seo, J. Y.; Kim, H.-J.; Pai, S. J.; Do, X. H.; Yoon, H. G.; Hwang, S. S.; Han, S. S.; Baek, K.-Y., Facile control of defect site density and particle size of UiO-66 for enhanced hydrolysis rates: Insights into feasibility of $\mathrm{Zr}$ (IV)-based metal-organic framework (MOF) catalysts. Appl. Catal. B 2019, 245, 635-647. 